Research Article

\title{
Decision System for Plural Production Lines Layout by Using GA
}

\author{
Hidehiko Yamamoto ${ }^{1, *}$, Masato Noda ${ }^{2}$, Hirohumi Tsuji ${ }^{3}$, Yasuhisa Terawa ${ }^{3}$, Yoshinori Nakamura ${ }^{3}$, \\ Masayuki Tsuchida ${ }^{3}$, Katsuaki Yamada ${ }^{4}$, Yukiyasu Kuriyama ${ }^{4}$ \\ ${ }^{1}$ Department of Mechanical Engineering, Gifu University, Yanagido 1-1, Gifu, Gifu 501-1194, Japan \\ ${ }^{2}$ KIOXIA Corporation, Shibaura 3-1-21, Tokyo, Japan \\ ${ }^{3}$ New Business Development Div., InfoFarm Co., Ltd, Yanaizu-cho Distribution Center 1-8-4, Gifu, Gifu 501-6123, Japan \\ ${ }^{4}$ Production Div., Kai Industries Co., Ltd, Oyana 1110, Seki 501-3992, Japan
}

\section{ARTICLE INFO}

Article History

Received 23 October 2020

Accepted 08 April 2021

Keywords

Line layout

genetic algorithm

Chameleon-code

production line

walking time

\begin{abstract}
We develop the system to decide the efficient layout of assembly production line by using Genetic Algorithm (GA). We call the system as Decision System of Plural Production-lines-layout by GA (PPG). PPG decides the efficient layout of production line by using GA, work-flow-line acquired by Chameleon code and the machine breakdown data. PPG evaluates the layout efficiency by calculating the operator's walking time to fix the machine breakdown occurred on the production line. By the evaluation, it is ascertained that PPG was useful.
\end{abstract}

(c) 2021 The Authors. Published by Atlantis Press B.V. This is an open access article distributed under the CC BY-NC 4.0 license (http://creativecommons.org/licenses/by-nc/4.0/)

\section{INTRODUCTION}

We need the efficient production line layout. In this study, Decision System of Plural Production-lines-layout by Genetic Algorithm (PPG). PPG determines the layout of plural production lines where some operators work. The determination is carried out by using Genetic Algorithm (GA) and the operators' walking routes obtained from Chameleon-code. PPG is applied to the razor assembly line to verify whether PPD is effective in improving work efficiency. The efficiency means which kind of layout that plural production lines are working just one operator can manage.

\section{CHAMELEON CODE}

Chameleon code is the new color barcode whose function has a high speed and precision recognitions as shown in Figure 1. This study puts Chameleon-code on the top of an operator's hat and obtains the operator's walking routes data by many cameras that are put on the factory ceiling. The acquired operators' walking routes data includes the name of the operator, the camera names, the dates and times, and the $x$-and $y$-coordinates of the chameleon code.

\section{PPG CHARACTERISTICS}

Plural production-lines-layout by genetic algorithm is the system to determine the layout of plural production lines using GA. The

Corresponding author.Email: yam-h@gifu-u.ac.jp determination is based on the operators' walking routes data which are acquired from the chameleon-code and the machine breakdown data. Specifically, PPG calculates the walking time that an operator moves to fix machine breakdowns and determines the better layout where one operator can manage plural production lines. As shown in Figure 2, PPG consists of the condition module and the GA module. Condition module includes the acquired operators' walking routes data and various parameters for GA. GA module determines the better production line layout by using GA.

\section{INDIVIDUAL REPRESENTATION}

The individual representation of our GA used in PPG adopts the structural gene, as shown in Figure 3. We express the $x$-coordinates, the $y$-coordinates and the angles indicating the position of one production line as the three consecutive loci. When representing plural production lines, these three loci are connected in series. Figure 3 shows the individuals in the case of three production lines. The $x$ coordinates, $y$-coordinates and angles are shown in Figure 4 . To secure the moving paths of the operator, we adopt the constraint that the width between each production line is an arbitrary value $Z \mathrm{~mm}$. The $Z$ means the width that an operator can walk through. Individuals that do not satisfy the constraints are deleted as lethal genes.

\section{HOW TO EVALUATE FITNESS}

The GA fitness of PPG adopts the walking time of an operator. If the walking time of individual $i$ is $t_{i}$ and the slowest walking time 

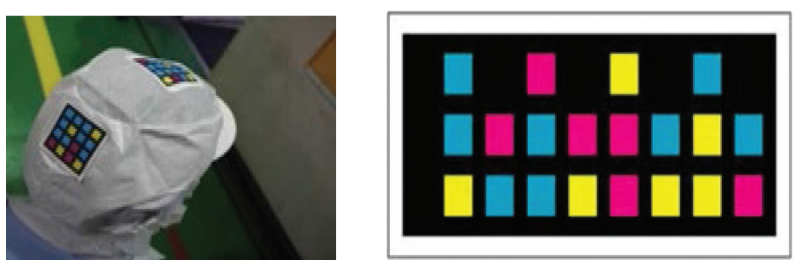

Figure 1 Chameleon-code.

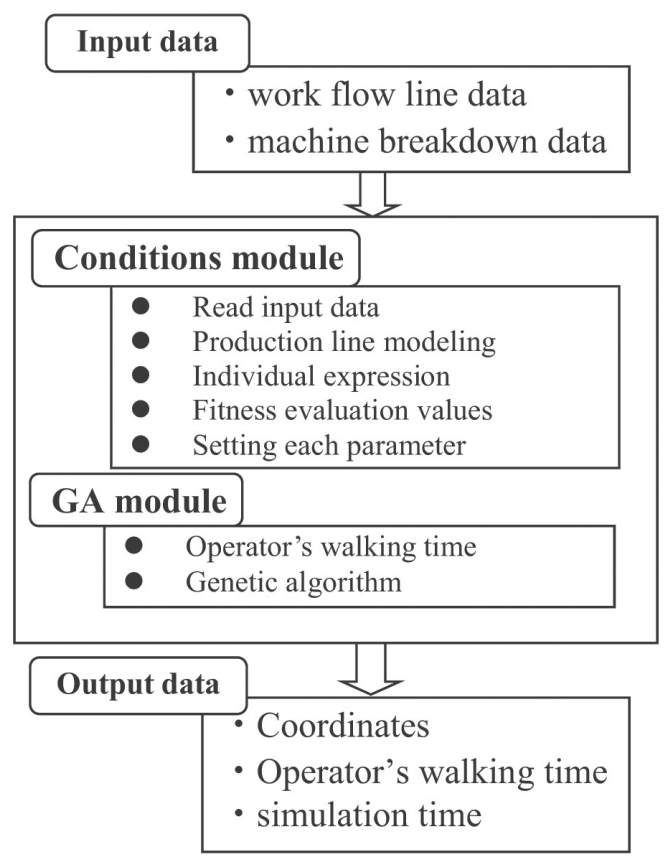

Figure 2 PPG outline.

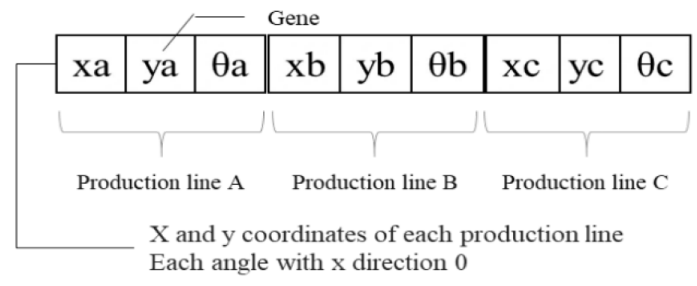

Figure 3 Individuals expression.

among the population of a generation is $t_{w}$, the fitness $f_{i}$ of individual $i$ is expressed by the following equation.

$$
f_{i}=\left(t_{w}-t_{i}+1\right)^{2} \quad(i=1,2,3, \ldots)
$$

If the time difference between each individual and the slowest individual is adopted as the fitness, the difference between an excellent individual fitness and another individual one becomes small. To make large difference, we give the difference a square [1].

\section{GA ALGORITHM}

Our GA module function is to improve the efficiency of production line layout by GA. The algorithm of our GA is shown below.

Step 1: Generate the initial population.

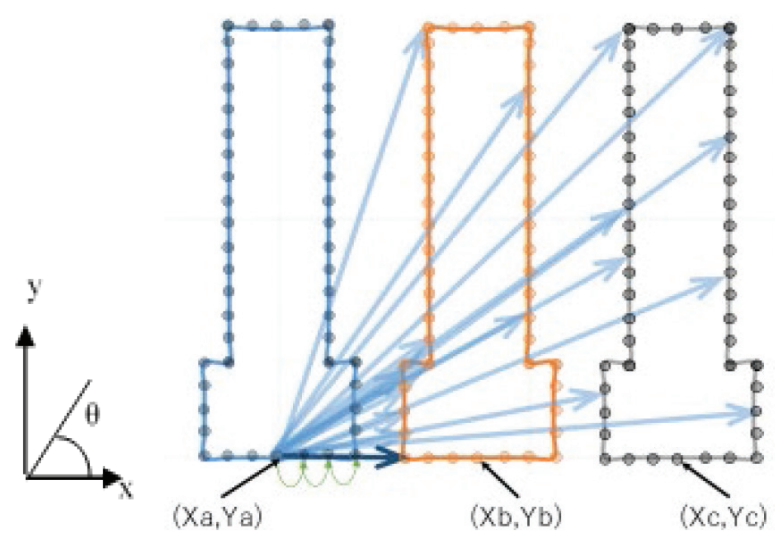

Figure 4 How to judge lethal chromosome.

Step 2: Calculate the machine breakdown coordinates for each production line.

Step 3: Calculate the walking time of the operator.

Step 4: Calculate the fitness.

Step 5: Judge whether the end condition is satisfied or not. If satisfied, go to Step 8. If not, go to Step 6 .

Step 6: Carry out selection, crossover and mutation operations and generate new individuals of the next generation.

Step 7: Carry out Step 2 on the new generation's individuals.

Step 8: The production line layout of the individual with the shortest walking time is adopted as the satisfying layout and is output as a solution.

We describe the details of the above algorithm as below.

Step 1: Generate the initial population.

The individuals of the initial population are randomly selected from among the spaces coordinates that lines can be placed in the factory. The information of the selected individual has the $x$ - and $y$-coordinates and angles of the production line. In generating the individuals, there is a possibility that the production line layouts may overlap or be too close. In this case, the individual corresponding to these kinds of layouts becomes a lethal gene. Not to generate the lethal genes in generating individuals, the following operations are carried out.

(1) Whenever generating individuals, calculate the distance between the contour of a production line and the contours of other still acquired production lines as shown in Figure 4. (2) If the calculated distance is less than $Z \mathrm{~mm}$, the individuals are deleted as a lethal gene. (3) Return to (1) and regenerate the individual again.

Step 2: Calculate the machine breakdown coordinates for each production line.

The coordinates whose machine gets breakdown in the factory layout change according to the coordinates of the production line layout. That is, since the coordinates of a machine breakdown occurrence for every generated individual are different, it is necessary to calculate each walking time. PPG's coordinates of machine breakdown occurrences of all individuals are calculated by the following operations. 
(1) Find the work flow line data corresponding to the machine breakdown occurrence time from the machine breakdown data and the coordinates of the machine breakdown. (2) By using the acquired coordinates, calculate the machine breakdown occurrence coordinates for other all individuals. For example, if the coordinates and angles of one production line A of an individual generated in Step 1 are $\left(x_{a}, y_{a}, \theta_{a}\right)$ and the machine breakdown coordinates found in (1) are $\left(x \_e, y \_e\right)$, the machine breakdown coordinates $\left(X_{e}, Y_{e}\right)$ of the production line $A$ are expressed as the following equations.

$$
\begin{aligned}
& X_{e}=x_{a}+x_{e}{ }^{*} \sin \theta_{a} \quad(e=1,2,3, \ldots) \\
& Y_{e}=y_{a}+y_{e}{ }^{*} \cos \theta_{a} \quad(e=1,2,3, \ldots)
\end{aligned}
$$

Step 3: Calculate the walking time of the operator.

When a machine breakdown occurs, the operator moves from the operator's standing place to the place where the machine breakdown occurs to fix it. PPG calculates the total walking time to get there and back.

Step 4: Calculate fitness by using Equation (1).

Step 5: Judge whether the end condition is satisfied or not. If satisfied, go to Step 8. If not, go to Step 6.

After the walking time of the operator is updated, if no better individual is acquired after certain generations, GA is finished.

Step 6: Carry out selection, crossover and mutation operations.

Using the fitness calculated in Step 4 and using the fitness proportional method, select a set of the individuals as a crossover target. As the crossover, we adopt the two-point crossover. Mutations occur with a certain probability.

Step 7: Carry out Step 2 on the new generation's individuals.

Repeat Steps 2-6 for the next generation population.

Step 8: The production line layout of the individual with the shortest walking time is adopted as the satisfying layout and PPG outputs it as a solution.

The coordinate data of the solution individual is output and the best layout is represented by using the acquired coordinates.

\section{APPLICATION EXAMPLES}

Plural production-lines-layout by genetic algorithm was applied to the razor assembly line of Kai Industries Co., Ltd. Since the shape of the razor assembly line is extremely complicated, we simplified it to the graphic model as shown in Figure 5 and applied it to PPG.

In this case, we acquired the operator walking routes data for $8 \mathrm{~h}$ per one shift. The routes data correspond to 3 days. The machine breakdown data of the same time (Table 1) are also acquired. We adopted the GA parameters as follows. The mutation rate is $10 \%$, the population size is 200 individuals and one individual is preserved as an elite. Selection and crossover methods were carried out by the fitness proportional method and two-point crossover. We also adopted $b=500 \mathrm{~mm}$ and $Z=800 \mathrm{~mm}$.

The simulation results are shown below. The simulations were carried out 20 times. The acquired best layout is shown in Figure 6. The layout corresponds to the individual with the minimum

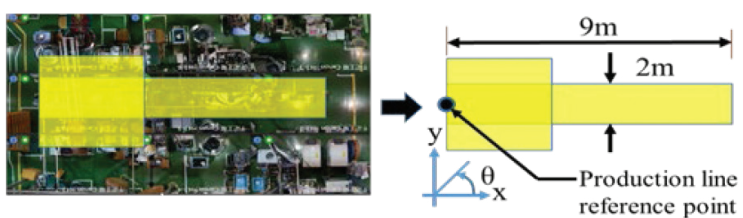

Figure 5 Production line model.
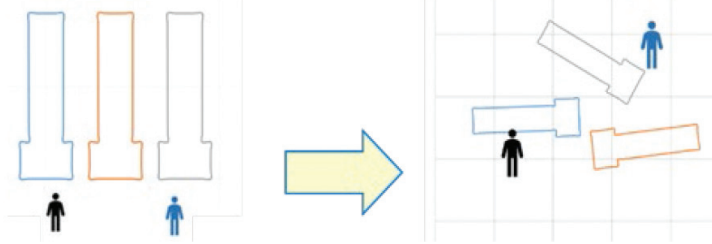

Figure 6 Parallel layout and PPG layout.

Table 1 Machine breakdown example

\begin{tabular}{lccl}
\hline Time & $\boldsymbol{x}$-Coordinate & $\boldsymbol{y}$-Coordinate & Breakdown name $\boldsymbol{A}$ \\
\hline 411 & 3513 & 4046 & Whether the blade is abnormal \\
565 & 3858 & 2046 & Whether the blade is abnormal \\
890 & 4128 & 881 & $\begin{array}{l}\text { On standby } \\
\text { Abnormal presence or absence } \\
1156\end{array}$ \\
2651 & 3468 & of frame \\
\hline
\end{tabular}

Table 2 Comparison of simulation results

\begin{tabular}{lc}
\hline & Walking time (s) \\
\hline PPG layout & 2405 \\
Parallel layout & 6164 \\
\hline
\end{tabular}

walking time. As a comparison, the walking time of the current layout adopted in Kai Industries Co., Ltd. Its current layout is arranged in parallel. The current layout walking time was also calculated. The layout determined by PPG shortened the operator walking time by about 63 min compared to the parallel layout (Table 2). The layout determined by PPG was effective in improving work efficiency.

\section{CONCLUSION}

After application simulations, it is ascertained that PPG using the chameleon code of InfoFarm Co., Ltd. is effective for the decision to find better layouts of plural production lines where one operator works. We will need the more research to find the refined layout of plural production lines because the acquired layout is not beautiful.

\section{CONFLICTS OF INTEREST}

The authors declare they have no conflicts of interest.

\section{REFERENCE}

[1] K. Honda, H. Yamamoto, T. Yamada, Design system of cell type assembly machine with dual arms robot by GA, Artif. Life Robot. 23 (2018), 170-173. 


\section{AUTHORS INTRODUCTION}

\section{Dr. Hidehiko Yamamoto}

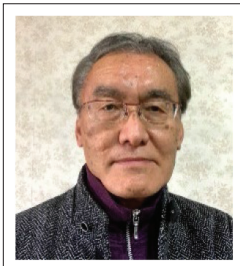

He received his B.S, M.S., and $\mathrm{PhD}$ from Nagoya Institute of Technology. After joining Toyota Industrial Corporation and Wakayama University, he works for Gifu University. He is currently a Professor and the fellow of the Japan Society of Mechanical Engineers.

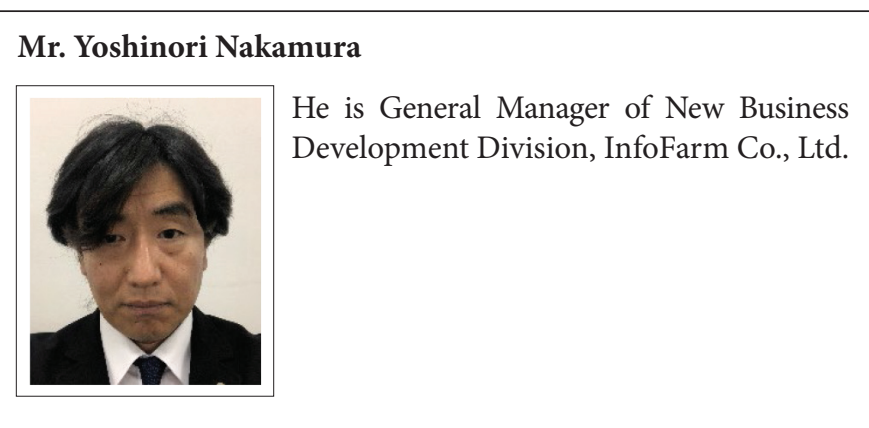

Mr. Masayuki Tsuchida

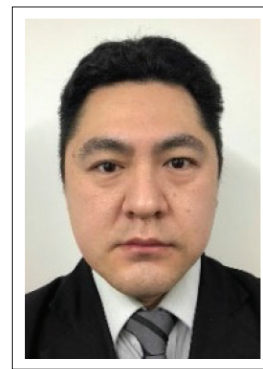

He is Expert of New Business Development Division, InfoFarm Co., Ltd.

\section{Mr. Hirohumi Tsuji}

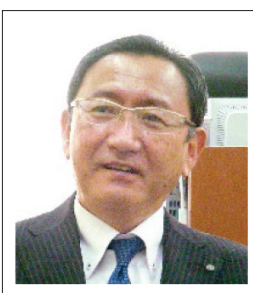

$\mathrm{He}$ is President and Representative Director of InfoFarm Co., Ltd.

\section{Mr. Yasuhisa Terawa}

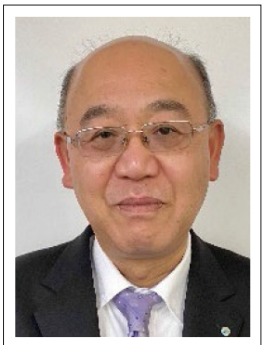

He is Senior Expert of Corporate Planning Department, InfoFarm Co., Ltd.

\section{Mr. Yukiyasu Kuriyama}

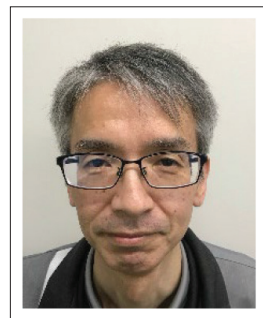

He received his B.S. from the Department of Mechanical Engineering, Nippon University, 1986. Currently, he is a Chief Manager Technical Def., Prod. Div. Kai Industries Co., Ltd. 